\title{
Sustainable Design in Construction Sector
}

\author{
L. Bragança \& R. Mateus \\ University of Minho, Department of Civil Engineering, Guimarães, Portugal
}

H. Koukkari

VTT Building and Transport, Building Construction Technology, Espoo, Finland

\begin{abstract}
Evaluation of the sustainable development - and on the other hand the effects of human activities on the future of the nature and mankind - is made on the basis of various indicators. The choice of indicators, the use of indicators and the evaluation procedure comprise both objective and subjective issues. The results of a sustainability evaluation procedure will be better interpretative and understandable when decision-making theories are applied. This concerns especially the valuation and use of weighting-factors that link the measurable and objective parameters of indicators. When the most important indicators are generally agreed, the differences between cases, countries or technological branches can be handled through weightingfactors. Further, dealing with the performance criteria of the built environment in relation to the sustainability can be advanced by application of decision-making methods.
\end{abstract}

\section{INTRODUCTION}

Building construction affects each four topics of sustainable development that are ecological, economic, social and cultural development. The fundamental goal of sustainable development is to preserve the ecological systems that globally are the basis for human life and biodiversity of the nature. The European Commission has initiated a working group "Competitiveness of the Construction Industry". According to this group, the challenges of the construction sector are:

- environmentally friendly construction materials

- $\quad$ energy efficiency of buildings

- construction and demolition waste materials

- water conservation

- $\quad$ health in buildings

- building related transport aspects

- urban sustainability

- societal impacts arising from construction activities and from the built environment.

The policy-makers and decision-makers need reliable information on the current state of sustainable topics and future influences of human activities that is obtained on the basis of the best knowledge of the art. However, problems of fundamental nature still need to be solved in search of general acceptance of sustainable assessment methodologies. Arguments of scientific uncertainty have been used in objection of environmental taxes (Jenkins et al 2002). Major reasons for difficulties are the political, technological and cultural differences of countries. They are also caused by the dependence of a subjective valuation involved in each general methods developed so far.

Decision-making theories and practices have been recently used to overcome the imminent gaps between the goal of explicit results and the entanglement of basic values. Again, different approaches have been used. The novel methods do not withdraw the subject involvement but they help to understand its relation to different results. 


\section{SUSTAINABILITY EVALUATION WITH INDICATORS}

\subsection{Development of sustainability indicators}

Several countries follow the development of sustainability by the aid of indicators that cover the different topics. An indicator is usually a value derived from a combination of various parameters. A parameter is a measurable or observable property, which provides information about a phenomenon/environment/area with a significance extending beyond that directly associated with a value. Indicators have to be defined in a clear, transparent, unambiguous and right way, even before the concern whether they relate and evaluate several parameters. After this, it can be indispensable to define the areas of evaluation and the respective parameters.

The indicators are in general defined according to their nature: driving force (pressure), state and response. Different indicators have been developed by administrations, organizations and industries at local, national and global levels. In addition, different parameters and their observation and assessment methods are in use. At the European level, the following ten indicators are defined by a Working Group nominated by the Commission in 1991:

\begin{tabular}{|lll|}
\hline core indicators & - & satisfaction of citizens \\
& - & impact on the climate change \\
& - & movability and traffic services \\
& - & access to services and green areas \\
- supplementary indicators & - & quality of air \\
& - & distances to school \\
& - & management systems of sustainable development \\
& - & noise \\
& - & sustainable land use \\
& - & products according to sustainable development
\end{tabular}

Use of sustainability indicators and parameters is based on definitions, rules, methods, classifications and weighting. In most of these phases, valuation and rating made by individuals are incorporated either in the development or in use of the methodology.

\subsection{Sustainability indicators and eco-efficiency in the construction and real estate sector}

The sustainability indicators give information on the influences of the construction and real estate sector as a whole as well as on the impacts of planning, design, ownership and use phases of a building. They may be used in evaluation of a building, enterprise, sector or even a simple construction product, expressed by the aid of parameters. There exist different approaches to develop and use the indicators due to the local character of the sector and differences of societies, environment and geography.

The indicators and accordingly the parameters are organized according to environmental, functional, economic and social criteria, often the two latter ones being combined. According to the European co-operative project CRISP, the Sustainable Development issues are:

1 Environmental: Natural raw materials including use of water. Bio-diversity. Energy. Environmental pollution. Land use.

2. Economic: Economic development and finance; indicators dealing with costs, productivity profitability. Production and consumption; indicators describe the quantity or quality of production or consumption. Urban and community services and responses; indicators dealing with economic responses etc.. 


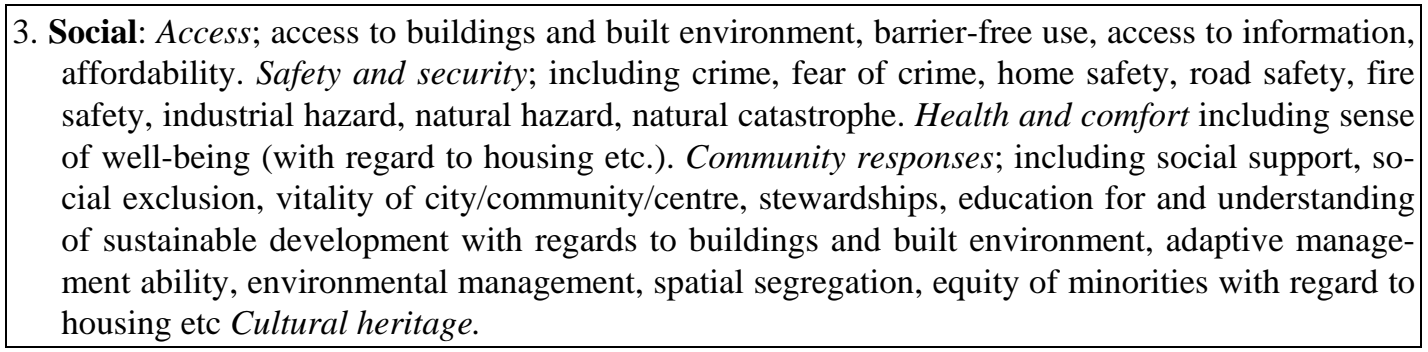

Eco-efficiency is related to the sustainable development, especially to the environmental and economic topics. According to OECD, eco-efficiency expresses the efficiency with which ecological resources are used to meet human needs. When speaking about challenges of Factor 10or Factor 4- development, it is meant the goal to increase the eco-efficiency in industrial and development countries, respectively. Eco-efficiency is defined with terms that have different qualities. In order to get any value for the eco-efficiency, the different indicators of the input and output have to be analyzed and combined. The methods used in the LCA may be adopted.

Indicators of eco-efficiency are not yet well established or practically experienced (Häkkinen et al 2002). Proposals have been made both for principles to define the indicators and their subjects. The methodologies are debated and developed in several research institutes currently.

\subsection{Environmental Life Cycle Analysis}

The construction and real estate sector has great responsibility for the change of the ecological systems as it:

- consumes natural resources (materials, energy, water);

- produces waste and emissions (e.g. greenhouse gases, sewing water);

- takes land and forestry into civil use.

The Life Cycle Analysis (LCA) is an evaluation procedure of the environmental effects of any kind of product, process or activity, from the cradle to grave (figure 1). The LCA is generally accepted to be applied for construction products, too. This analysis is made by identification, what has been taken from the environment and what has been brought back, by assessment of the potential harms due to these actions and by rating the significance of the impacts. The rating (or valuation) part of the LCA varies from country to country, and it is often neglected.

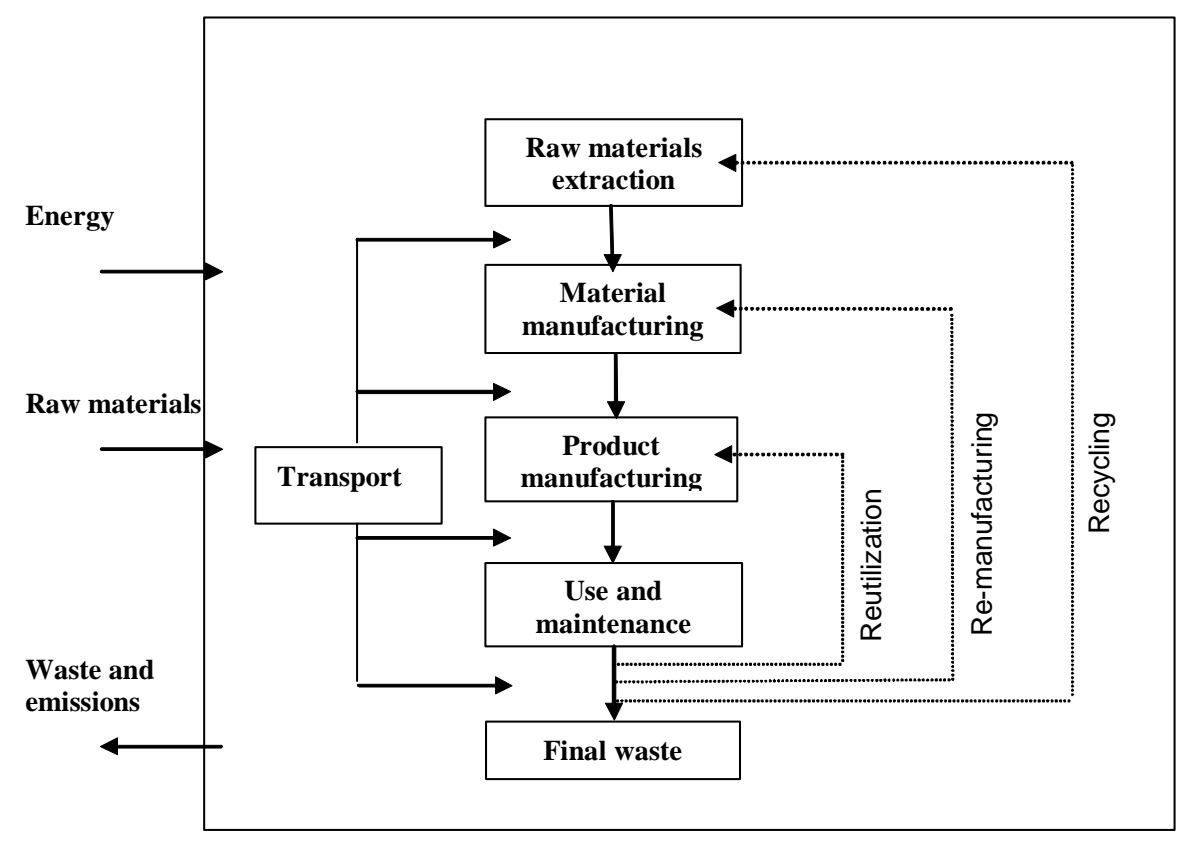

Figure 1. Life Cycle of a product. 
The LCA includes three main phases that are inventory, analysis of potential effects and the interpretation (valuation) of the results (figure 2). The inventory phase includes identification and quantification of the consumption of energy and materials and the gases emitted to the environment. It can be completed by assessing the potential environmental effects that are climatic warming, acidification, eutrophication (excess thriving of aquatic flora), formation of photochemical oxidants, loss of ozone, harmfulness to health and ecotoxicity.

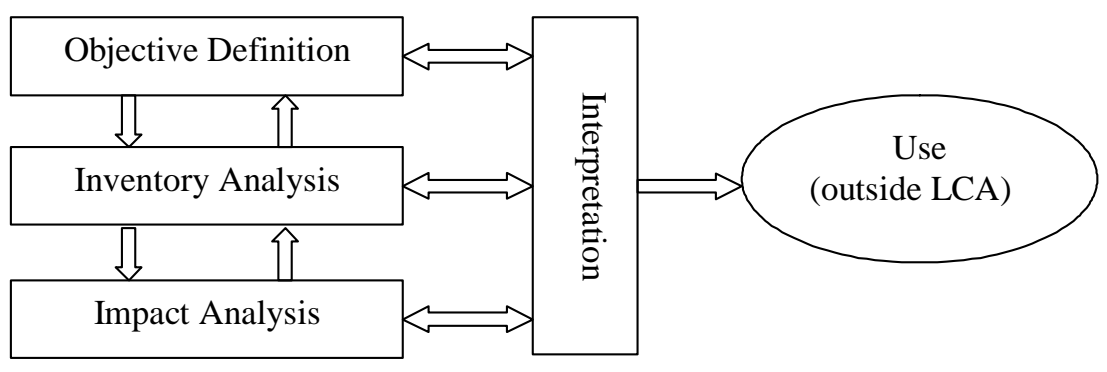

Figure 2. Phases of the LCA.

Even though the framework of environmental analysis has achieved international unanimity, there is no detailed instruction or agreement about the method. This means that environmental analyses have been, and can be, carried out with varying boundaries and principles of valuation, which affects the result. Kaipiainen and Häkkinen (1996) emphasize that the principal endeavor of the whole methodology is the quantification of the result, even though they may also contain qualitatively described components. The major problem of the result is the multitude of figures, if the results have not been combined by valuation, and its openness to interpretation.

\section{DEVELOPMENTS ON THE EVALUATION METHODS}

\subsection{General}

The relation of the various indicators shall be developed after the evaluation of each indicator in analysis. This relation is normally established giving an equal importance to all the indicators. The choice may be not the most correct one once the indicators are not expressed in the same order of magnitude and/or in the same unit. For example, the contribution of a material for the greenhouse effect is presented in the amount of carbon dioxide emitted, the acidification in equivalent of hydrogen ions, the electro fission in nitrogen equivalent, etc. On the other hand, the way that each parameter influences the sustainability is neither consensual nor unalterable along the time. So, it is difficult to express the sustainability of a solution in absolute terms, through an indicator that integrates all of the analyzed parameters and that allows the quantitative classification of a solution's sustainability.

Some systems and tools for the sustainability assessment are being implemented or in the development phase. Its application is complex and needs the previous knowledge of some data. Some of the sustainability assessment tools have datasheets that gather some of the needed data, although the data is related with the particular aspects of the country of origin, which turns its application in a different country very difficult.

In most methodologies and tools of sustainability evaluation, the functionality aspects of construction solutions are forgotten. However, it is practically impossible to compare two distinct construction solutions that present exactly the same performance at all levels of the functional parameters, due to the existing limitations of the materials standard dimensions and their physical properties. It is also commonly known that with small concessions at the level of the economical and environmental performances it is possible to increase significantly the overall performance of a building, what significantly contributes for improvement of the solution's sustainability. On the other hand, the use of the functional indicators on the sustainable assessment is an attempt to prevent errors from the past, where the concept "sustainable solution" has been associated to construction solutions with good environmental performance, but without fulfilling the necessary functional requirements (comfort, durability, etc.). 
The results of the assessment particularly depend on the analysed indicators and in the weight considered for each indicator.

\subsection{Evaluating the environmental performance}

Interpretation (valuation) of information may be done according to the standard ISO 14042. It presents methods to analyze and assess the data concerning the emissions. The first step is to categorize the parameters identified in the inventory phase of the LCA based on their causeconsequence relations. Steps to calculate the indicator factor of each impact category includes determination of weight factors. The weight attributed to each indicator is given based on the following criteria: spatial scale of the impact, severity of the hazard, degree of exposure and risk for being wrong. With the knowledge of a qualitative evaluation it becomes necessary to convert the evaluation into a quantitative scale.

Internationally, the interpretation of the results of the LCA is under rapid development. The methods of decision-making with decomposition and synthesis are in general applied in recent developments of the interpretation and valuation of the environmental indicators. In search for generally accepted indicators, it seems that the development leads to different weighting factors in different countries (Häkkinen et al 2002).

In Finland, the Decision Analysis Impact Assessment (DAIA) has been used to categorize the emission effects on atmosphere and waters. The categories to be considered are climate change, acidification, creation of ozone in the lower levels of atmosphere and eutrophication (excess thriving of aquatic flora). In the DAIA and other methods for rating, the factors also develop due to updating of knowledge on the real effects.

In Portugal, the development of the interpretation and valuation phase of the LCA is an example of adoption of the EPA's list (EPA, 2000), presented on Table 1, and application of an multi-criterion methodology of analysis that is based on the theory AHP (Analytic Hierarchy Process) presented by Saaty (1990).

Table 1. Weight of environmental impacts according to EPA's list. The categories in the Finnish application are shown with *.

\begin{tabular}{ll}
\hline Impact category & Current consequences \\
\hline Global warming* & \multicolumn{1}{c}{ Low } \\
Acidification* & High \\
Eutrofphication* & Medium \\
Fossil fuel depletion & Medium \\
Indoor air quality & Medium \\
Habitat alteration & Low \\
Water intake & Medium \\
Criteria air pollutants & High \\
Smog & High \\
Ecological toxicity & Medium - Low \\
Ozone depletion* & Low \\
Human health & Medium - Low \\
\hline
\end{tabular}

Through an AHP process as comparison pair to pair (Pairwise Comparison Value), the numerical comparison is attributed to each one of the possible pairs in the list of the qualitative values. Thus we can determine the number of times that the weight of a parameter must be higher than another one and establish a relation between all the parameters in study (Table 2).

A quantitative evaluation for 8 and 12 parameters is presented in the following table 3 . The presentation of these two classifications is linked to the possibility of not having all the data needed in the 12 parameters evaluation, being only possible the 8 impacts evaluation. 
Table 2. Pairwise Comparison Value.

\begin{tabular}{ll}
\hline Verbal importance comparison & Pairwise comparasion values \\
\hline Highest vs. Low & 6 \\
Highest vs. Medium & 3 \\
Highest vs. High & 1.5 \\
High vs. Low & 4 \\
High vs. Medium & 2 \\
Medium vs. Low & 2 \\
\hline
\end{tabular}

Table 3. Evaluation of impacts.

\begin{tabular}{lll}
\hline Impact category & $\begin{array}{l}\text { Current consequences } \\
8 \text { impacts (weight \%) }\end{array}$ & $\begin{array}{l}\text { Current consequences } \\
12 \text { impacts (weight \%) }\end{array}$ \\
\hline Global warming & 24 & 16 \\
Acidification & 8 & 5 \\
Eutrofphication & 8 & 5 \\
Fossil fuel depletion & 8 & 5 \\
Indoor air quality & 16 & 11 \\
Habitat alteration & 24 & 16 \\
Water intake & 4 & 3 \\
Criteria air pollutants & 8 & 6 \\
Smog & & 6 \\
Ecological toxicity & & 11 \\
Ozone depletion & & 5 \\
Human health & & 11 \\
\hline Total & 100 & 100 \\
\hline
\end{tabular}

\subsection{Evaluating the functional performance}

The analysis and comparison of the performance of construction solutions has to be carried out at the level of each element (interior walls, exterior walls, floor, roof, etc.), therefore each one of them present distinct requirements. The first step for the evaluation is to define functional indicators and parameters. The six essential requirements and durability according to the Construction Products Directive form a regulated basis for consideration, e.g. thermal insulation, airborne and impact sound insulation, flexibility of natural illumination, structural stability, air permeability, etc.

The quantification of these indicators is relatively simple according to various proven methods. However, the way each indicator influences on the performance and, therefore, the sustainability of a solution is not consensual. The evaluation involves subjective rating and depends above all on the type of use of the solution, as well as on socio-economic and cultural heritage of the subject.

In a first phase it can be considered that all functional indicators have the same weight in the evaluation of the functional performance. In order to obtain more consensual values it can be made interviews to the potential users in order to identify which indicators are considered more important. Through the application of a Multi-attribute Decision Analysis, p.e. the Analytic Hierarchy Process methodology, is possible to quantify the weight of each one of the indicators. Synthesizing all the functional indicators in one number it's possible to obtain the functional performance of the solution.

\subsection{Evaluation of the economic performance}

There are several costs associated to the life cycle of a building or material/construction system: costs of the materials (it includes extraction of raw materials, production, transport for the construction place), costs of construction, costs of utilization, costs of maintenance, costs of reha- 
bilitation, cost of demolition and costs of devolution to the natural environment, recycling or of reutilization.

Measuring the economic performance of a building is more straightforward than measuring the environmental performance. Standardized methodologies and quantitative published data are readily available.

Life-cycle cost analysis (LCCA) is a method for assessing the total cost of a facility ownership. It takes in account all costs of acquiring, owning, and disposing of a building or building system. LCCA is especially useful when project alternatives that fulfill the same performance requirements, but differ with respect to initial cost and operating costs, have to be compared in order to select the one that maximizes the net savings.

Thus, it's relatively easy to foresee the total costs associated to the life cycle of a building. As less will be the costs foreseen for a construction solution, better it will be the economical performance and more sustainable will be the solution.

In order to have a complete economical performance, the analysis of the costs associated to the solution's life cycle must be made, including the residual value. The residual value of a system (or component) is its remaining value at the end of the study period, or at the time it is replaced during the study period. Residual values can be based on value in place, resale value, salvage value, or scrape value, net of any selling, conversion, or disposal costs. As a rule of thumb, the residual value of a system with remaining useful life in place can be calculated by linearly prorating its initial costs. For example, for a system with an expected useful life of 15 years, which was installed 5 years before the end of the study period, the residual value would be approximately $2 / 3(=15-5 / 15)$ of its initial cost.

As it's well known, the construction solutions are very distinct at the level of the durability. It is essential to use the same study period for each alternative whose LCCs are to be compared according to the stakeholder perspective. For example, a homeowner would select a study period based on the length of time he or she expects to live in the house, whereas a long-term owner/occupant of an office building might select a study period based on the life of the building.

\subsection{Balancing the environmental, functional and economic performance}

After comparing the solutions in each group of indicators (environmental, functional and economic) it is necessary to classify globally the sustainability of the solution. The way as each group of indicators influences the sustainability is not consensual. So, it is acceptable, in a first step, that the three groups of indicators present the same weight.

The experience shows that the most compatible alternatives with the environment are generally the most expensive. However, considering that with the implementation of the concept "sustainable construction" is intended a bigger compatibility between the artificial and the natural environments, without compromising the functional performance, easily it's understood that the weight of the environmental and functional indicators must be higher than the weight of the economic indicators in the sustainability evaluation. The Equation 1 shows how the three groups of indicators could be balanced, in order to determine an absolute value (sustainable score - SS), that expresses the sustainability of a solution (Bragança et al, 2004).

$$
S S=W_{1} x \sum_{i=1}^{m} W_{E n p(i)} x E n p_{(i)}+W_{2} \cdot \sum_{i=1}^{n} W_{F p(i)} x F p_{(i)}+W_{3} \cdot \sum_{i=1}^{o} W_{E c p(i)} x E c p_{(i)}
$$

where, $S S$ = sustainable score; $W_{1}=$ weight of the environmental parameters' group; $W_{E n p(i)}=$ weight of the environmental parameter (i); $E_{n} p_{(i)}=$ value of the environmental parameter (i); $W_{2}$ = weight of the functional parameters' group; $W_{F p(i)}=$ weight of the functional parameter (i); $F p_{(i)}=$ value of the functional parameter (i); $W_{3}=$ weight of the economic parameters' group; $W_{E c p(i)}=$ weight of the economic parameter (i); $E c p_{(i)}=$ value of the economic parameter (i); $m$ = number of environmental parameters under analysis; $n=$ number of economical parameters under analysis; and $o=$ number of economical parameters under analysis. 


\section{CONCLUSIONS}

Sustainable design, construction and use of buildings are based on the evaluation of the environmental pressure, functional aspects (related to the users and the local building codes) and life-cycle costs. There is an environmental effect when something is taken from the environment as a resource or returned to it as waste or emissions, which weakens or threatens the availability of resources, the livable environment and the human health. The sustainable design searches a bigger compatibility between the artificial and the natural environments without compromising the functional requirements of the buildings and the costs associated.

The accomplishment of a LCA provides an excellent support tool for the decisions making, however, its necessary much time and money due to the necessary amount of information. One of the limitations of the tool is that it does not guarantee that a company who uses it has its products relatively more "environmental-friendly" than others, therefore can not be used as marketing. In the international scene, the responsible entities for the technical standards, from which the world guides its production, has been extremely presented. Among them it is distinguished ISO (International Organization for Standardization), in which it detaches norm ISO 14000 that is one of the most excellent tools of environmental management.

With the knowledge of the weights of each of the environmental indicators, it is possible to determine the value of a unique environmental indicator. With the knowledge of the economic indicators, functional and even the socio-cultural, and the application of the multi-criterion analysis methodology, it is possible to determine a unique global value for evaluation of the sustainability. Future developments on the sustainable assessment methodologies should bring more consensual lists of indicators to be evaluated and more consensual weighting factors.

\section{REFERENCES}

Bragança, L. \& Mateus, R. 2004. Sustainability Assessment Datasheet. COST C12 - Final Report.

Häkkinen, T. \& Kaipiainen. M. 1996. Ecological criteria in building planning. Helsinki: Building Information Institute. 52 p. (In Finnish).

Häkkinen T. et al. (2002) Eco-efficiency in the building and real estate sector. Helsinki: Ministry of Environment, Housing and Building Department, the Finnish Environment 580. 165 p.

ISO 14042:2000 - Environmental Management - Life Cycle Assessment - Life Cycle Impact Assessment: establishes the guidelines for the Life Cycle Impact Assessment of a LCA study.

Jenkins R. et al. (2002). Environmental regulation in the new global economy, The impact on industry and competitiveness. Glos, UK: Edward Elgar Publishing Ltd. 349 p.

Saaty, T. L. 1990. How to make a decision: The analytic hierarchy process. In the European Journal of Operational Research, Vol. 48, No. 1, pp. 9-26.

United States Environmental Agency (EPA), Science Advisory Board (SAB), 2000. Toward integrated Environmental Decision Making. EPA-SAB-EC-00-011, Washington, D.C.. 